\title{
Arrival Time Estimation and Reminders on Public Bus System
}

\author{
Bo-Wei Lin ${ }^{1}$, Ding-Ray Huang ${ }^{2}$, Yun-Chung Yang ${ }^{3}$, Yi-Chin Huang ${ }^{4}$, Ye Chin Kiong ${ }^{\#}$, Jim-Min \\ Lin and Ming-Fong Tsai* \\ Department of Information Engineering and Computer Science, Feng Chia University, Taichung, Taiwan \\ Industrial Ph.D. Program of Internet of Things, Feng Chia University, Taichung, Taiwan
}

\begin{abstract}
The public bus service, which is one of the most frequently used public transportation methods, is an indispensable part of public lifestyle. Hence, government and companies have integrated the public bus service with smart device applications, enabling the public to check information such as bus schedules and instant information at any time. Their aim is to improve the efficiency of the public bus service. Unfortunately, their efforts have faced a variety of problems, such as the distrust of the public regarding the provided information, traffic situation, and unexpected incidents which disturb the bus schedule. In order to overcome the mentioned problems, a smart bus stop signboard system will be designed. The smart signboard will show the current position of the bus and provides a reservation service, which the public may access using smart devices. The proposed system allows bus drivers to obtain information about a particular bus stop to determine whether they should drive to that bus stop. Because the traffic situation and the embarkment and disembarkment of passengers affects the arrival time of the bus, the proposed system will take the change in the number of bus passengers and travel time into account in order to predict the arrival time of the bus.
\end{abstract}

Keywords: Public bus service, arrival time, predict.

\section{Introduction}

The public bus system plays an important role in connecting locations within a city, completing the whole transportation system of cities. A city with many modes of transportation is still incomplete without the presence of public buses. In this modern era, population increases in both cities and rural areas have also led to an increase in vehicles. When traffic becomes heavy, the efficiency of the public bus system - which also reduces the number of personal vehicles on the road - becomes a significant issue to be improved. In some areas, the bus frequency is low and the public has no choice but to wait for a long period of time. However, a long waiting time discourages the public from taking the bus [1]. In urban areas, the public often prefer personal vehicles, taxis, and even Uber services over bus services because they cannot predict the arrival time of public buses. Due to this discouraging situation, the number of cars and motorcycles on the road cannot be decreased efficiently and the goal of introducing an efficient public transportation system is not achieved. Thus, in order to remedy the situation, the improvement of the public bus system is a critical issue [2]. A few bus stops in Taichung City introduced LED screens which show predicted arrival times of the buses. Smart device applications have also been developed so that the public can check the bus arrival time when needed. However, the provided predictions are not accurate. Situations have been observed when the arrival time is given as three minutes, but the actual waiting time exceeds the predicted time. This situation causes public anxiety while waiting and can even lead to individuals missing the bus [3]. Because this situation leads to distrust of the public bus system, the public still prefer other transportation methods

\footnotetext{
+ Corresponding author: Ming-Fong Tsai

E-mail address: cn90111@gmail.com, alex@alexleo.click, a5502008@gmail.com,oppi22@gmail.com, yechinkiong@gmail.com, jimmy@fcu.edu.tw and tsaimf@fcu.edu.tw*
} 
over public transport. It has been noticed that the preciseness of predicted arrival times affects the utilization rate of the public bus system. Thus, in order to encourage the utilization of the public bus system, an improvement of arrival time prediction is necessary.

\section{System Structure}

One of the factors is certainly the traffic situation. Another factor is the flow of passengers, which is inconsistent. The higher the number of passengers getting on or off the bus, the greater the time spent at each stop. Hence, we can conclude that the number of passengers is a variable factor. If we are able to acquire instant information on the traffic situation and passenger numbers and then modify the predicted arrival time based on this information, an optimized prediction can be determined. In order to acquire data about the two mentioned factors, this paper proposes the introduction of a smart device application and smart bus stop signboards. The smart device application and smart bus stop sign work together to acquire the passenger count at each stop. The bus route traffic situation is retrieved periodically. The data will then be evaluated at the server which will process a modified arrival time prediction as shown in Figure 1.

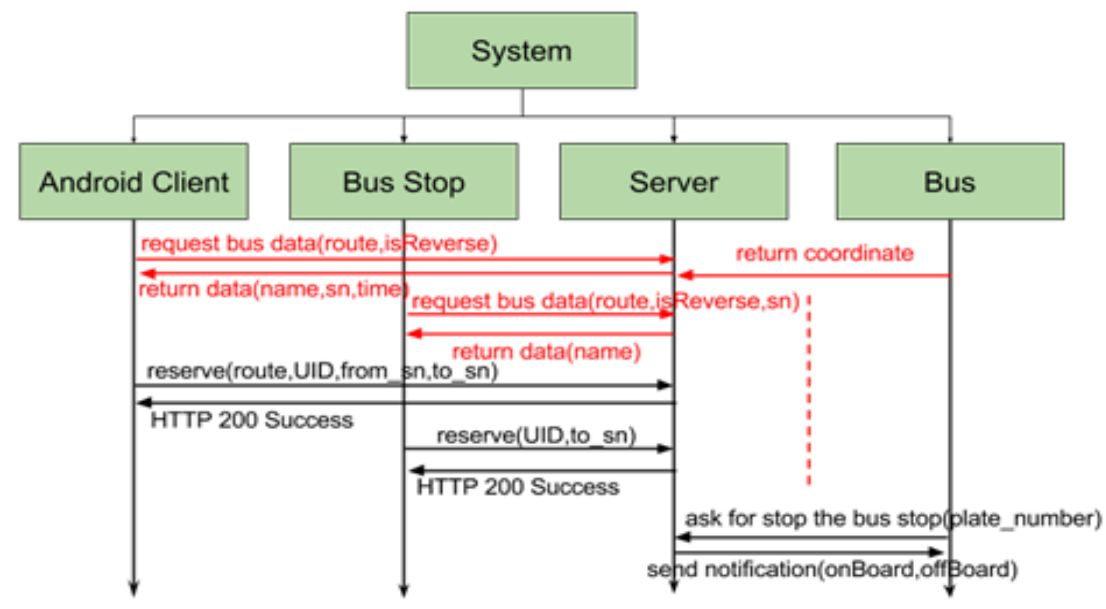

Fig. 1: System structure diagram

\subsection{Correction Method}

This section explains the method of making corrections to the bus arrival time as shown in Equation 1 . Algorithm 1 represents the proposed correction formula. We assume that the bus arrival time is T, whereas $\mathrm{T}$ ' represents the corrected arrival time. The number of total passengers at the particular bus stop is $\mathrm{X}$. The average time is represented by $\mathrm{N}$ (in seconds) for a passenger to get on or off the bus. Using Algorithm 1, the arrival time prediction will be corrected from stop to stop.

$$
\mathrm{T}^{\prime}=\mathrm{T}+\mathrm{X}^{*} \mathrm{~N}
$$

\subsection{Correction Method}

The server will consistently retrieve public data about public buses from the Taichung City Government unit. Besides, Google API will be used to estimate the travel time based on distance and the current traffic situation. The information will be stored in the server database. On the other hand, the user reservation data, which is collected by the client application (smart device application), will be processed using Algorithm 1 along with cached information. The processed result will be provided to the client side through HTTP API, such that the client device may acquire the corrected result. The client site refers to the smart device and smart bus stop signboard. The presence of the client application enables the server to acquire the reservation counts at each bus stop. The reservation data contributes to an accurate time prediction when Algorithm 1 is adapted. First, the GPS location preciseness of each bus stop will be corrected in the local database, at the same time acquiring location information at each stop using Taichung City Government's open data. Reservation data for each stop will be collected through smart device applications and smart bus stop signboards. The estimated arrival time of the bus will be acquired through Google API. When these data are computed using Algorithm 1, the correct estimation will be stored in the database and cached such that the client site can access the required route estimation time instantly. 


\subsection{Smart Device Application}

Smart device application is the medium for the proposed system to collect data. Two functions are provided in the application: check bus arrival time, bus service reservation. To avoid abuse of the system, the user is required to register for an account before accessing the reservation service. After logging into the system, the user is able to select the starting station and the terminal and then press the reservation button to complete the reservation process. After a reservation is made and once the bus is approaching, the application will vibrate the smart device as a notification. This will prevent the user from missing the bus. When the bus arrives at the terminal station, the smart device will also vibrate. Aside from notifying the user, the reservation system provides data to the server for evaluation of passenger numbers. When the application starts, the user is able to select a route. After selection, the dynamic information of the respective bus will be shown. Before proceeding to the reservation process, the system will check whether the user had logged into the system. If not, the system will prompt the user to login. When the user is in the blacklist - which means that the user violated a rule such as reserving a bus but not showing up - the user is not able to use the reservation service. If the user account is valid, reservations can be completed as shown in Figure 2.

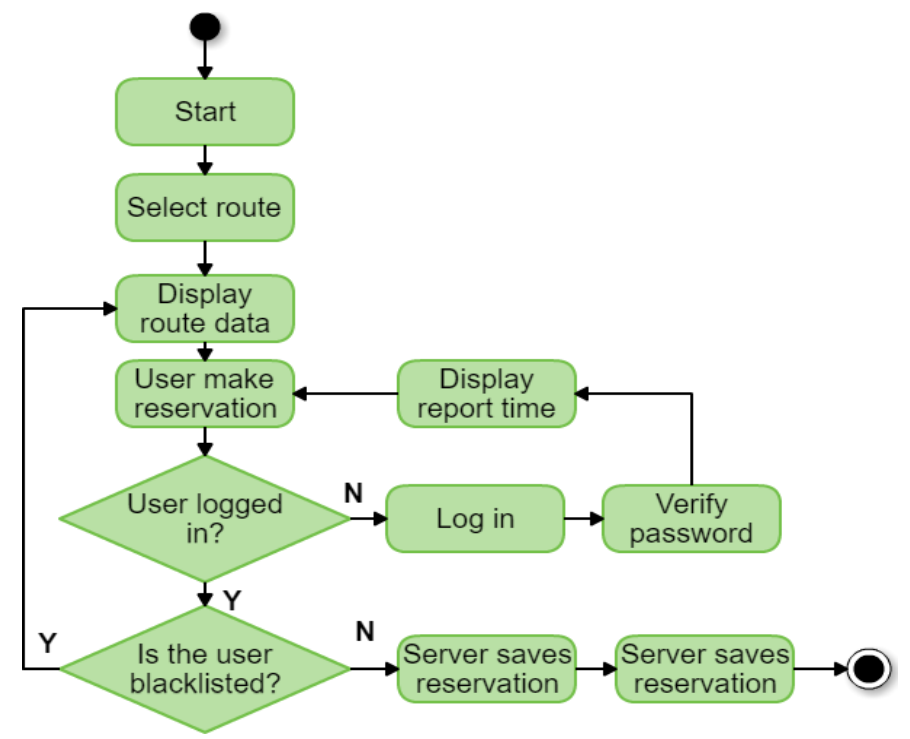

Fig. 2: Reservation process flowchart

\subsection{Smart Signboard}

Although most people have a smart device on hand, there is still a group of people who don't have a smart device. There are also people whose smart devices do not connect with the mobile network. These people are potential users of the bus system, yet they are unable to make reservations in the system or check the bus status using smart devices. This situation will cause the information accumulation process to be incomplete and Algorithm 1 cannot perform optimally with inaccurate data. To avoid the mentioned problem, a smart bus stop signboard will be designed to collect data from users who do not access the proposed system through smart devices. The basic function of the smart signboard is indifferent to the smart device application. The signboard will show the approximate location of the bus. In addition, during the reservation process, the user need not select the starting point, because the starting point will be automatically set as the current stop. In order to avoid abuse of the system, the user will be required to perform verification through an e-mail address. When the smart signboard is activated, it will request bus information from the server and display the information. Next, it will check on whether the reservation button has been pressed. If a card has been swiped, the system will determine whether the action succeeded. When the process succeeds, reservation data will be sent to the server. Otherwise, the system will inform the user of the failure and then continue to request bus information from the server to show the latest information as shown in Figure 3. 


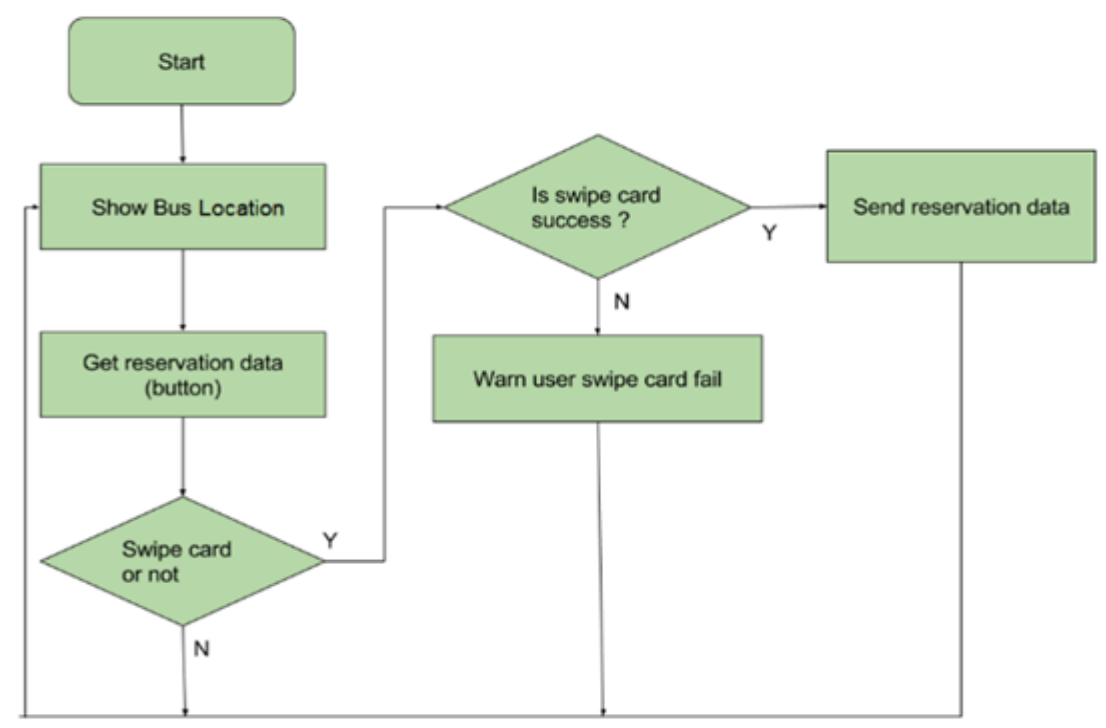

Fig. 3: Smart signboard flowchart

\subsection{Bus Site}

The reservation data collected through the smart device application and smart signboard do not just provide information for the correction algorithm. The data is also provided to the respective bus through another smart application designed for the bus site. The feature is quite simple. When the bus approaches the bus stop, if there are reservations from the public, the application will inform the bus driver to stop at the bus stop. Traditionally, when no passenger rang the bell to get off the bus at a particular stop, the bus driver would observe whether people raise their hands at the stop, which is a gesture that implies one wishes to take the bus. However, there are circumstances when the bus driver might not notice a person's gesture, causing the person to miss the bus. Now, the notifying feature ensures that the bus approaches the stop when needed. If the bus approaches the stop due to the notification and the driver discovers that nobody is getting on, the bus driver has the ability to report the situation to the server. Figure 4 shows the flow diagram of the bus site application. When the bus driver boots up the application, the system gives a prompt for bus ID input. The bus ID will help the system to identify the location and route of the bus. When the bus appears near to a stop, the system will notify the bus driver to approach the stop if reservation data is detected. At the same time, if the bus driver discovers that nobody at the stop wants to take the bus, the bus driver can report to the server. The system will verify whether the bus is at the reported stop before sending the report to the server.

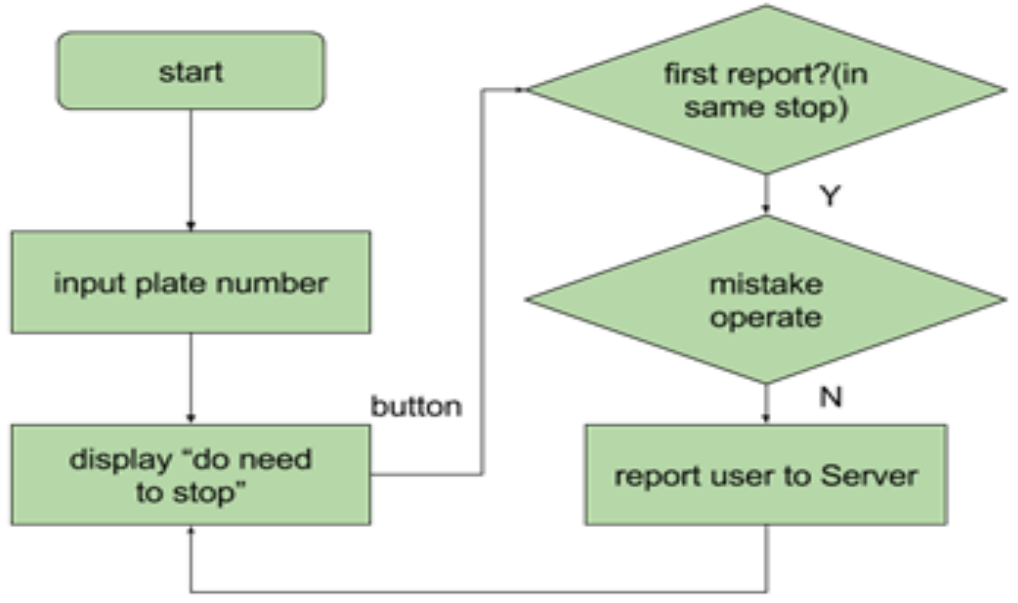

Fig. 4: Bus site application flowchart

\section{Data Collection and Result Analysis}

In this paper, the analysed route was the Taichung Route 5, from Feng Chia University (Fu Xing Road) stop to Maple Garden Stop. The reason for choosing this route is that it has a wide passenger flow. As the system is still in the developmental stage, instead of acquiring reservation data through applications and 
smart signboards, the passenger flow was recorded manually. The recorded data includes: estimated arrival time from government open data, actual arrival time, time, and the number of passengers getting on the bus and getting off the bus. The bus route, position, and direction are provided by the Taichung City Government. The travel time between each stop was acquired through Google API. The collected data includes 56 bus trips on the selected route. Twenty-three trips out of 56 were during peak hours (17:00 19:00). Among the 56 trips, 30 trips were recorded from the Feng Chia University stop to the Maple Garden Stop; the remaining 26 trips were the reverse route, from Maple Garden to Feng Chia University. The smart device application runs in the Android system environment. Figure 6 shows the interface of the implemented result. In Figure 5(a) and Figure 5(b), a login button can be seen. Users can press the button to log in. When the Routes button in 5(a) is pressed, the application switches to a screen as shown in Figure 5(b), where the user is able to select a route to check on the status. After route selection, the route information is shown as in Figure 5(c). The estimated arrival time at each stop is shown. The distance between the bus and the stop is differentiated with colours: red, orange, yellow, and green. Red implies that the bus is nearest to the stop, and so on. To make a reservation, the user can simply press the reserve button found at the bottom left corner. A notification will pop up when a reservation is made, as shown in Figure 5(d). A reservation can be cancelled by pressing the cancel button.

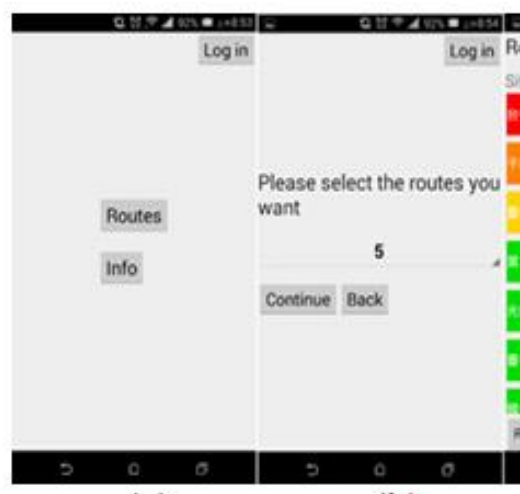

(a) (b)

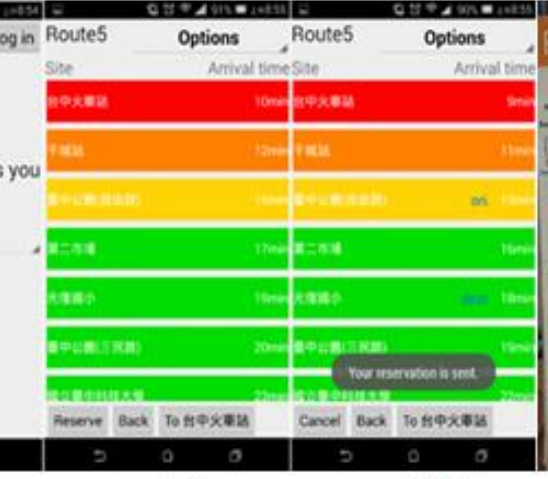

(c) (d)

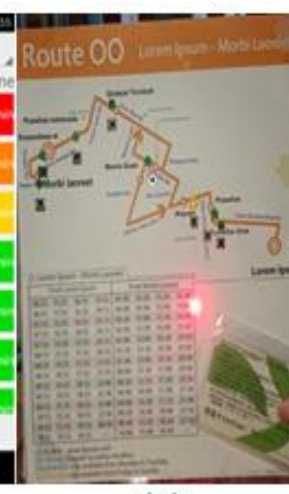

(e)

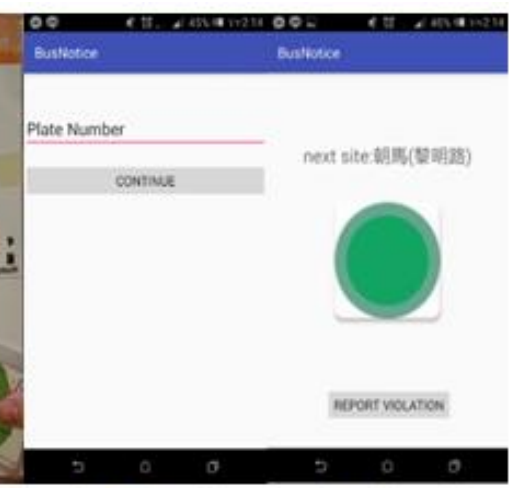

(f)

Fig. 5: Android application

The smart signboard will be developed using the Arduino Mega module. It utilizes the Ethernet network module to send and receive data. The RFID module is used to acquire e-ticket information upon reservation. Figure 5(e) shows the developed smart signboard. The LED light on the board implies the approximate location of the bus. The upper right corner is the e-ticket detection area. If the ticket (card) is successfully detected, a red LED will blink. To make a reservation, the user simply presses the destination point and then scans the e-ticket. The process is simple and it gives access to every user. The bus side application serves to detect reservation requests and notify the bus driver to approach a bus stop. It reduces the burden on the bus driver on deciding whether to approach a bus stop and also avoids circumstances when a passenger misses the bus due to the driver's incorrect decision. As in Figure 5(f), the bus driver is required to input the route ID number. After the route ID number is confirmed, the system proceeds to Figure 5(g). The name of the next stop will be shown. If the round figure is green, it means that no reservation data has been found at the next stop. If it turns red, that means that someone made a reservation and the driver should approach the stop. If the driver approaches the stop and finds out that nobody is there, the driver can press the "report violation" button to inform the server. Our interpretation shows that the average time for each passenger to get on or off the bus is 2.4 seconds. This value is fed into Algorithm 1. The outcome of the corrected time is compared to the open data arrival time and the actual arrival time, and is shown in Figure 6. Figure 6(a) and Figure 6(b) are the results of random bus trips during the same time period. According to Figure 6 , it is noticeable that the corrected arrival time is relatively close to the actual arrival time when compared to the predicted arrival time provided by government open data. The graphs prove that the proposed algorithm is capable of performing an efficient correction. In Figure 6(c) and Figure 6(d), the 0 axis implies the actual arrival time. The red lines are the predicted arrival time produced using Algorithm 1 and the green line is the raw arrival time provided by government open data. According to Figure 6, in both off-peak hours and peak hours, the red lines are closer to the 0 axis, which means that our algorithm is efficient during both time periods. Figure 6(e) shows the precise data comparison between our deviation values and open data deviation values. Among the results produced by our system's algorithm, $74.2 \%$ of these are more accurate than the open data results. 
Of the buses, $56.04 \%$ arrive earlier than the time predicted by government open data, causing the users to miss buses. When our algorithm is implemented, the proportion drops to $23.63 \%$, which means $32.41 \%$ of the chance of the user missing the bus is eliminated.

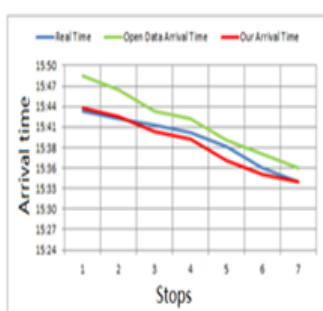

(a)

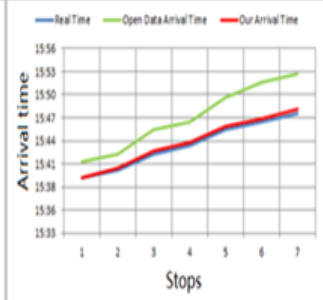

(b)

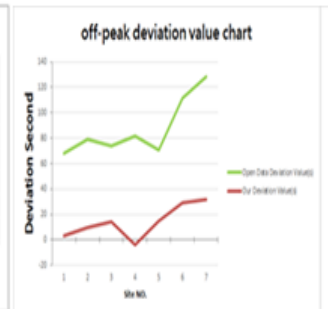

(c)

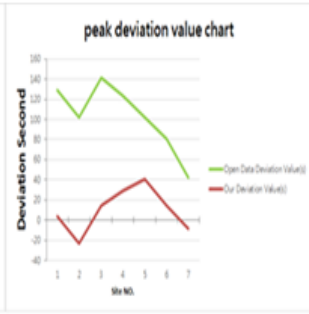

(d)

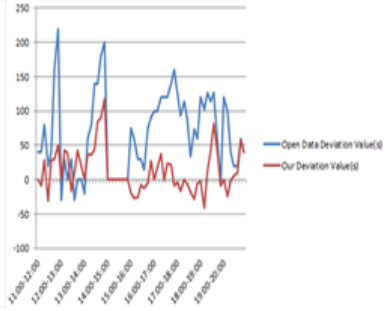

(e)

Fig. 6: Comparison between actual arrival time, open data arrival time, and our arrival time; Comparison between off-peak hours and peak hours; Comparison of the timeline chart

\section{Conclusion}

In this paper, the proposed algorithm successfully predicted an arrival time which is relatively close to the actual arrival time. In the future, we will seek to improve the algorithm such that more variables can be taken into account; for example, acquiring the bus route traffic-light programme from the intelligence transportation system and retrieving the bus driver's driving route records. With improvements, we believe that a more precise predicted arrival time can be computed using this algorithm.

\section{Acknowledgement}

We thank the Ministry of Science and Technology of Taiwan for supports of this project under grant number MOST 105-2815-C-035-099-E, MOST 105-2221-E-035-065 and MOST 105-2622-E-035-008-CC3. We thank co-authors and reviewers for their valuable opinions.

\section{References}

[1] V. Silva, T. Sciammarella, M. Campista and L. Costa, Time of arrival prediction with dynamic route tracking for public transportation systems. IEEE Annual Mediterranean Ad Hoc Networking Workshop, pp. 1-8, 2015.

[2] F. Sun, Y. Pan, J. White and A. Dubey, Real-time and predictive analytics for smart public transportation decision support system. IEEE International Conference on Smart Computing, pp. 1-8, 2016.

[3] T. Zhu, F. Ma, T. Ma and C. Li, The prediction of bus arrival time using global positioning system data and dynamic traffic in-formation. IEEE Wireless and Mobile Networking Conference, pp. 1-5, 2011. 